\title{
Graphene as a local probe to investigate near-field properties of plasmonic nanostructures
}

\author{
Sören Wasserroth, ${ }^{1, *}$ Timo Bisswanger, ${ }^{1, \dagger}$ Niclas S. Mueller, ${ }^{1}$ Patryk Kusch, ${ }^{1}$ Sebastian Heeg,,${ }^{2,3}$ Nick Clark, ${ }^{3}$ \\ Fredrik Schedin, ${ }^{4}$ Roman Gorbachev, ${ }^{5}$ and Stephanie Reich ${ }^{1}$ \\ ${ }^{1}$ Institut für Experimentalphysik, Freie Universität Berlin, 14195 Berlin, Germany \\ ${ }^{2}$ Photonics Laboratory, ETH Zürich, 8093 Zürich, Switzerland \\ ${ }^{3}$ School of Materials, University of Manchester, Manchester M13 9PL, United Kingdom \\ ${ }^{4}$ National Graphene Institute, University of Manchester, Manchester M13 9PL, United Kingdom \\ ${ }^{5}$ School of Physics and Astronomy, University of Manchester, Manchester M13 9PL, United Kingdom
}

(Received 12 February 2018; published 17 April 2018)

\begin{abstract}
Light interacting with metallic nanoparticles creates a strongly localized near-field around the particle that enhances inelastic light scattering by several orders of magnitude. Surface-enhanced Raman scattering describes the enhancement of the Raman intensity by plasmonic nanoparticles. We present an extensive Raman characterization of a plasmonic gold nanodimer covered with graphene. Its two-dimensional nature and energy-independent optical properties make graphene an excellent material for investigating local electromagnetic near-fields. We show the localization of the near-field of the plasmonic dimer by spatial Raman measurements. Energy- and polarization-dependent measurements reveal the local near-field resonance of the plasmonic system. To investigate the far-field resonance we perform dark-field spectroscopy and find that near-field and far-field resonance energies differ by $170 \mathrm{meV}$, much more than expected from the model of a damped oscillator $(40 \mathrm{meV})$.
\end{abstract}

DOI: 10.1103/PhysRevB.97.155417

\section{INTRODUCTION}

Surface-enhanced Raman scattering (SERS) is a wellknown technique to enhance the intensity of inelastically scattered light [1]. It uses the plasmonic near-field of metallic nanoparticles or surfaces to enhance the Raman signal. SERS achieves enhancement factors of up to $10^{11}$ and enables the study of single molecules by light scattering [2-4]. A great enhancement is achieved using SERS substrates such as rough metal surfaces [5,6] or agglomerates of metal nanoparticles $[3,7]$. SERS is used in many applications, e.g., analytics, biosensing [8,9], and the preservation of artwork [10]. The fundamental process behind SERS is explained by different approaches, for example, the macroscopic electromagnetic enhancement theory [11,12]. Microscopic models are the optomechanical approach [13] and the treatment of SERS as higher-order Raman scattering [14]. While the electromagnetic enhancement theory describes the plasmonic enhancement as an external effect, microscopic approaches incorporate the enhancement of the Raman scattering process.

Common techniques to characterize the spatial distribution of the local near-field of a SERS hot spot are electron energy loss spectroscopy [15], scattering optical near-field microscopy (SNOM), and dual s-SNOM for uncovered particles [16-18]. The far-field resonance, in contrast, is often investigated by dark-field spectroscopy measuring the elastically scattered light [19]. It was shown that a gold dimer made of two gold nanodisks covered with suspended graphene can

\footnotetext{
*soeren.wasserroth@fu-berlin.de

†Present address: 2nd Institute of Physics, RWTH Aachen University, 52074 Aachen, Germany.
}

be used to study the enhancement of the local near-field as well as the local strain and doping [20-22]. Graphene is an excellent material for characterizing the local near-field by Raman scattering because of its constant Raman cross section and its two-dimensional (2D) character, which allows external forces such as strain to pull the graphene into the plasmonic hot spot [20,21,23]. Further, the Raman spectrum of graphene and its behavior under strain are well known [24,25]. The coupled graphene-nanodimer system has so far been characterized only at a fixed wavelength of $\lambda=638 \mathrm{~nm}[20,22]$. An in-depth study of its energy and polarization dependence remains missing.

In this paper, we determine the near-field properties of a gold dimer by measuring the Raman modes of suspended graphene. We use a canonical SERS system that consists of two gold disks forming a dimer with controllable dimensions. We determine the strain and doping of the suspended graphene in the plasmonic hot spot. The combined system of gold dimer and graphene shows a strong polarization dependence of the Raman intensity. For incident light polarization along the dimer axis a plasmonic hot spot forms between the gold disks, enhancing the integrated Raman intensity by $7 \times 10^{3}$. For polarization perpendicular to the dimer axis the gold disks act as single plasmonic particles yielding only weak enhancement. We measured the SERS enhancement as a function of the excitation energy and found the resonance maximum at $2.01 \mathrm{eV}$. We discuss the surprisingly large difference $(170 \mathrm{meV})$ between the plasmonic near-field and the plasmonic far-field resonances. Since graphene has no intrinsic resonance, all changes in the Raman intensity are attributed to the changes in the near-field enhancement of the plasmonic system. Atomic force microscopy (AFM) imaging and dark-field spectroscopy were performed to characterize the spatial and far-field properties of the plasmonic system. 

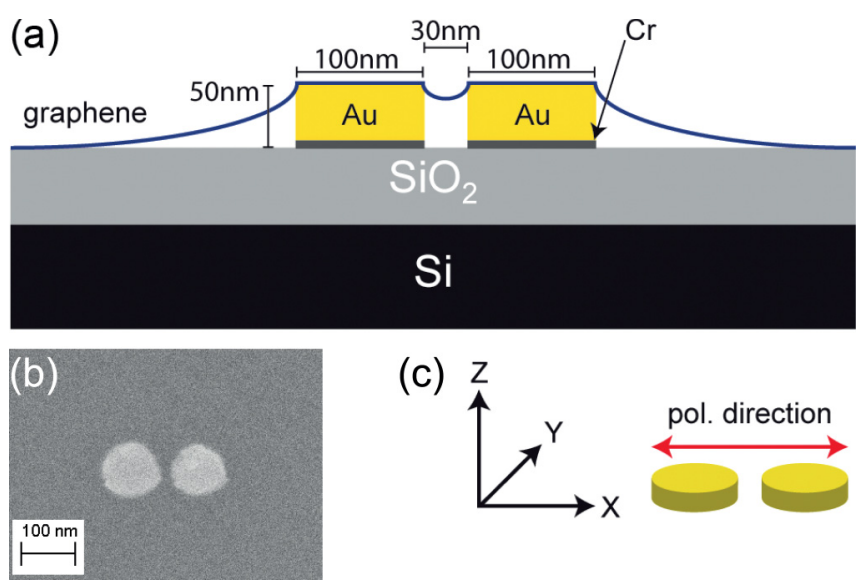

FIG. 1. (a) Sketch of the sample consisting of two gold disks covered with graphene on $\mathrm{SiO}_{2} / \mathrm{Si}$ substrate. The dimensions are indicated. (b) SEM image of a gold nanodisk dimer. (c) Coordinate system used throughout the paper. The polarization direction of the incident light was chosen along the dimer axis if not explicitly stated otherwise.

This paper is organized as follows. In Sec. II we describe the experimental setup and the sample geometry. Section III presents and discusses the results of the Raman and dark-field measurements. The local strain and doping of the graphene and the localization of the plasmonic hot spot are discussed in Secs. III A and IIIB. The polarization dependence of the Raman response of the dimer system is presented in the next part of the paper (Sec. IIIC). Section IIID presents the excitation-energy-dependent measurements of the near-field (Raman) and the far-field (dark field).

\section{EXPERIMENTAL PROCEDURE}

The sample consists of a field of plasmonic nanodimers that are built of two gold disks on a $\mathrm{SiO}_{2} / \mathrm{Si}$ substrate with a 290-nm-thick $\mathrm{SiO}_{2}$ layer [sketch in Fig. 1(a)]. A variety of dimer structures with different diameters was exposed by electron-beam lithography in a LEO 1530 Gemini FEG scanning electron microscope (SEM) and Raith Elphy Plus Lithography System with Laser Interferometer Stage. Cr (5 $\mathrm{nm})$ and $\mathrm{Au}(45 \mathrm{~nm})$ were evaporated, followed by a liftoff in an ultrasonic bath. The spatial properties of the system were imaged by (SEM; Zeiss ULTRA 55 with $10-\mathrm{kV}$ acceleration voltage) [see Fig. 1(b)]. A statistical evaluation of the SEM images of the investigated dimers yielded diameters between 100 and $110 \mathrm{~nm}$ and a gap of 20-30 nm.

A large flake of graphene was placed over the dimer field, covering multiple dimers. The graphene flake was prepared on a p-doped $\mathrm{SiO}_{2} / \mathrm{Si}$ substrate with a 90-nm oxide layer by micromechanical cleavage [26]. The flake was precisely transferred on top of the plasmonic structures. For the transfer the graphene was coated with a polymer supporting the socalled 'wet' transfer [27]. We checked by optical microscopy and Raman spectroscopy that monolayer graphene covered the region of the investigated plasmonic dimers [28]. The topography was measured by AFM using a Park Systems XE-150 in noncontact mode.
To access the near-field properties of the plasmonic system we measured the enhancement of the Raman modes of graphene as a function of the excitation energy. We use a tunable laser system to excite the plasmonic system in the wavelength range of 488-670 $\mathrm{nm}$ [29]. The light was focused and collected through a $100 \times$ microscope objective $(\mathrm{NA}=$ 0.9 ) with a laser spot size of $410 \pm 30 \mathrm{~nm}$ at an excitation wavelength of $602 \mathrm{~nm}$. Raman spectra were recorded with a Horiba T64000 spectrometer in backscattering configuration at room temperature. The positioning of the laser on the plasmonic hot spot is crucial, as small changes lead to a dramatic decrease or increase in intensity. To achieve maximum enhancement and comparable results in the excitation-energy-dependent measurements the laser focus was centered on the dimer with a piezo stage (PI E-710; step size, $100 \mathrm{~nm}$ ) for every measurement. The polarization of the laser was chosen to be parallel to the dimer axis unless specified otherwise [see Fig. 1(c)]. The laser power on the sample was kept constant at $300 \mu \mathrm{W}$. Calibration spectra of freestanding graphene were recorded at every wavelength to account for changes in the optical response of the setup. The enhanced intensity of the graphene modes in the excitation-energy-dependent measurements (see Sec. IIID) was divided by the intensity of the $\mathrm{G}$ and 2D modes of the calibration spectra of suspended graphene. Additionally, reference spectra of graphene were recorded on the substrate (without plasmonic nanostructure) $2 \mu \mathrm{m}$ from the dimer at every wavelength. The intensity of the Raman modes in the reference spectra was subtracted from the enhanced measurements to remove interference effects between the substrate and the graphene, ensuring that we investigate the plasmonic enhanced intensity [30].

The far-field resonance of the dimer was investigated by dark-field spectroscopy. The elastically scattered light was guided from the microscope with a $100 \times$ dark-field objective through a fiber to a Horiba Scientific iHR320 spectrometer. The sample was illuminated by a $50-\mathrm{W}$ white-light halogen lamp and the light was polarized along the dimer axis.

\section{RESULTS AND DISCUSSION}

We investigated the plasmon-enhanced Raman modes of graphene suspended on different dimers. Figure 2 shows that the transfer of graphene on top of plasmonic nanodimers yields a reproducible plasmonic enhancement at $633 \mathrm{~nm}$ for most of the dimers. Dimer VI shows no enhancement of the G and 2D modes, but a strong D mode is present; we conclude that for this dimer the graphene is torn in the gap, yielding no enhancement but defects. For the rest of the paper we focus on only one dimer (dimer I in Fig. 2) and characterize it in detail. For the investigated dimer system, strong enhancement and strain were reported previously at a 638-nm excitation wavelength with polarization along the dimer axis [20].

In Fig. 3 we show an exemplary plasmon-enhanced Raman spectrum of graphene in greater detail. One spectrum was recorded at a $605-\mathrm{nm}$ excitation wavelength while focusing on dimer I in Fig. 2 (black line in Fig. 3) and a reference spectrum was recorded next to the dimer on the substrate (blue). We labeled the prominent $\mathrm{D}, \mathrm{G}$, and 2D modes in graphene and their strain components $\mathrm{G}^{+}, \mathrm{G}^{-}, 2 \mathrm{D}^{+}$, and $2 \mathrm{D}^{-}[25,28]$. The reference spectrum shows only the unstrained $G$ and $2 D$ 


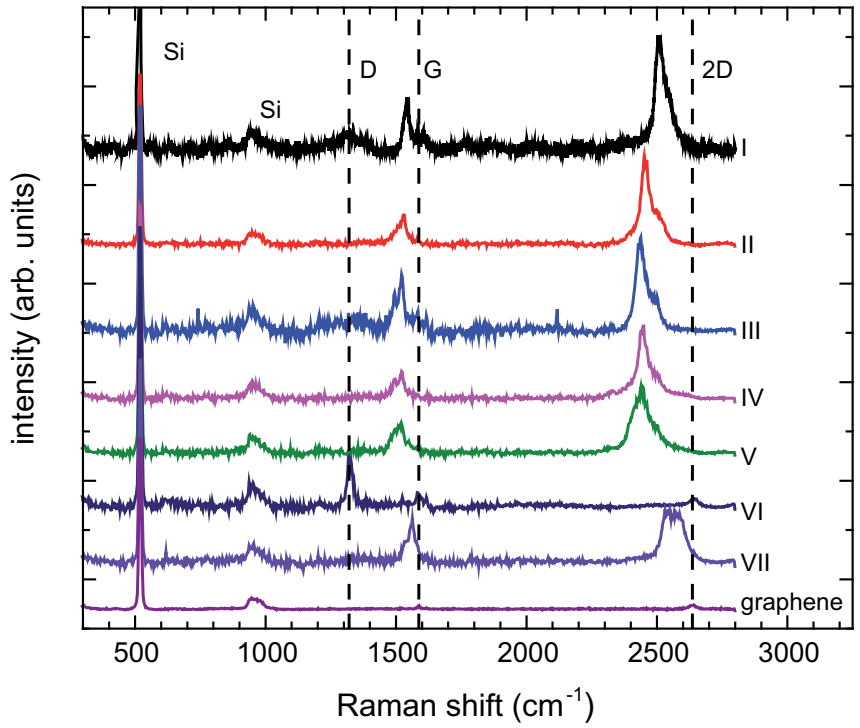

FIG. 2. Raman spectra of seven dimers (I-VII) showing plasmonic enhancement of the graphene $\mathrm{G}$ and 2D modes. It is compared to the bare graphene spectrum (purple). The silicon modes of the substrate are also shown. Spectra recorded at $633 \mathrm{~nm}$ and offset for clarity.

modes. We observe an increase in the integrated scattering intensity by a factor of 90 for the $\mathrm{G}$ and 55 for the 2D mode. The enhanced spectrum in Fig. 3 shows a shift and splitting of the $\mathrm{G}$ and the $2 \mathrm{D}$ modes with respect to the modes of the reference spectrum, which indicates that hydrostatic and shear strain as well as doping are present in the graphene sample (see Sec. III A) [22]. In addition to the enhanced and shifted modes the unstrained $G$ and $2 D$ peaks remain present as shoulders

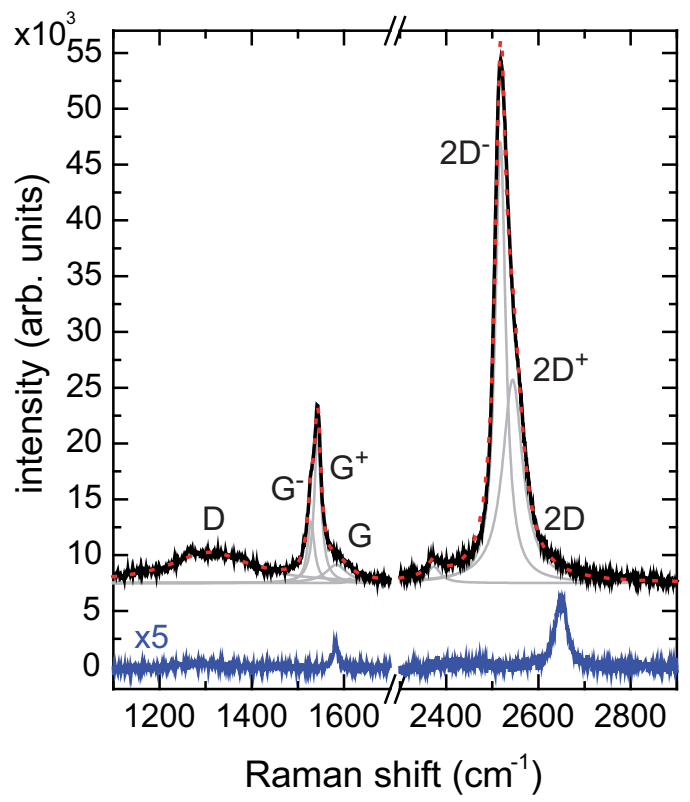

FIG. 3. Plasmon-enhanced Raman spectrum (black) when focusing on the nanodimer and a reference spectrum collected on the substrate (blue). The fitted peaks are shown in gray and the prominent Raman modes of graphene are labeled. Spectra offset for clarity. in the plasmon-enhanced Raman spectrum. These features originate from the graphene surrounding the dimer because the laser focus (diameter, $410 \mathrm{~nm}$ ) is much larger than the size of the plasmonic hot spot located in the gap of 30-nm size.

In Fig. 3 the D mode is shown in the plasmon-enhanced spectrum, whereas there is no indication of defect modes in the reference spectrum, as expected for high-quality mechanically exfoliated graphene. Activation of defect modes in plasmon-enhanced Raman scattering of graphene was reported previously and attributed to the spatial localization of the near-field [31]. Alternatively, the defect modes may originate from point defects in the cavity that are only visible when enhanced. The broad shape of the D mode is rather unusual for a defect-induced mode, consolidating the assumption of a plasmon-induced D mode.

A phenomenon often observed in SERS is the so-called blinking of the Raman modes [32]. It describes the sudden change in intensity of Raman modes over time. Figure 4 shows a series of Raman spectra of the G and 2D modes as a function of time. There is a small overall loss of intensity with time, presumably due to a shift in the sample position (defocusing). Neglecting this change, the intensities of the $\mathrm{G}$ and 2D modes remain constant, making them perfect candidates for evaluation of the integrated local field of the plasmonic system. In contrast, blinking occurs in the region of the $\mathrm{D}$ and $\mathrm{D}^{\prime}$ modes. These modes most likely depend on highly mobile and extremely localized features in graphene and/or in the plasmonic nanodimer such as mobile defects in graphene or mobile gold atoms [32]. The different behavior of the graphene Raman modes makes graphene an excellent material for testing plasmonic systems. The constant $G$ and $2 D$ modes allow investigation of the plasmonic resonance, whereas the blinking defect modes can provide more information about the confinement of the plasmonic near-field and will be the subject of future investigations.

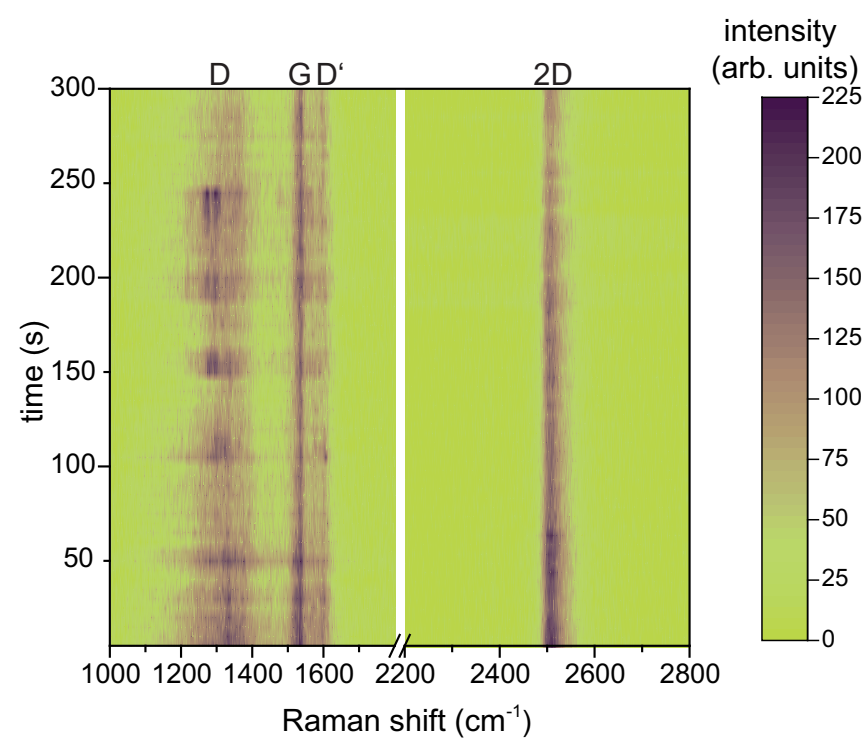

FIG. 4. Raman signal of the $\mathrm{G}$ and $2 \mathrm{D}$ modes recorded on the dimer over time. Each line shows the Raman intensity measured for $5 \mathrm{~s}$. Measurement was performed at a laser wavelength of $602 \mathrm{~nm}$. 

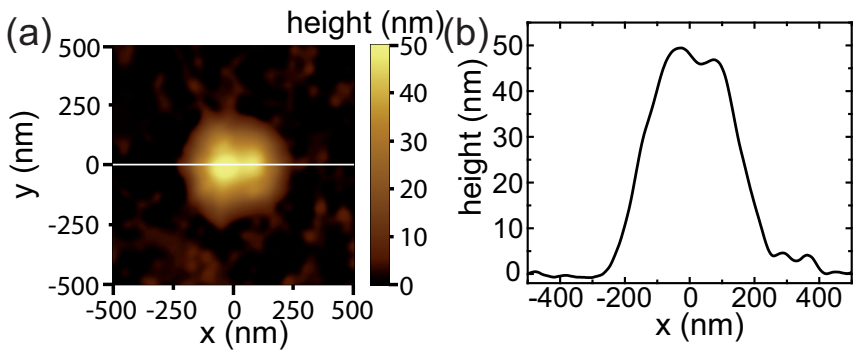

FIG. 5. (a) AFM topography image of the investigated dimer. (b) Height profile of the dimer at the position indicated by the horizontal gray line in (a).

\section{A. Local strain and doping}

The plasmonic enhancement of graphene Raman modes allows us to detect local properties of graphene in the hot spot at nanoscale resolution [20]. First, we evaluate the strain configuration and doping level for graphene suspended over a gold nanodimer. Tensile hydrostatic strain leads to a shift of the $\mathrm{G}$ and 2D modes to lower wave numbers. Shear strain induces a splitting of both modes [25]. Doping also results in a shift of the $\mathrm{G}$ and $2 \mathrm{D}$ modes due to the singular electron-phonon interaction for electrons at the Fermi surface (Kohn anomaly) [33]. Depending on the type of doping the modes shift to higher or lower wave numbers. For graphene in contact with gold nanoparticles n-type doping is characteristic [34,35]. It yields an upshift of the G-mode frequency, while the $2 \mathrm{D}$ line is almost constant in frequency for low doping levels, followed by a downshift at higher dopings [36].

The strain and doping level were determined with a correlation analysis of the $\mathrm{G}$ and $2 \mathrm{D}$ modes for arbitrary strain configurations [22]. For each Raman mode, we calculate the mean value of the two strain components $\overline{\omega_{\mathrm{G}}}=1534 \mathrm{~cm}^{-1}$ and $\overline{\omega_{2 \mathrm{D}}}=2512 \mathrm{~cm}^{-1}$ and obtain a hydrostatic strain of $2.1 \%$. This causes a $59-\mathrm{cm}^{-1}$ frequency shift of the $\mathrm{G}$ line. The shear strain amounts to $1.1 \%$ calculated from the $\mathrm{G}^{+}$and $\mathrm{G}^{-}$splitting of $7 \mathrm{~cm}^{-1}$. We find an n-type doping level of $10^{13} \mathrm{~cm}^{-2}$, corresponding to a shift of the $\mathrm{G}$ mode by $10 \mathrm{~cm}^{-1}$. These values report the local strain and doping of graphene in the dimer gap. According to the calculated near-field distribution in Ref. [20] the strongest plasmonic enhancement emerges in the dimer gap, leading to the conclusion that the SERS signal is dominated by contributions from the hot spot. The relative Raman intensities of the $\mathrm{G}^{+}$and $\mathrm{G}^{-}$components also allow evaluation of the lattice orientation of the graphene flake with respect to the dimer axis. The zigzag direction in the lattice of the graphene flake that covers the dimer is tilted by an angle of $9^{\circ}$ towards the dimer axis.

AFM measurements show the expected topography of graphene for local strain inside the dimer gap and around the dimer. The AFM topography image in Fig. 5(a) clearly shows the two gold disks and the graphene extending over the dimer. The graphene is pulled down towards the substrate due to adhesion forces straining the graphene in the gap between the disks, as shown in the height profile through the dimer center in Fig. 5(b). A detailed discussion of the strain configuration can be found in Refs. [20-22].
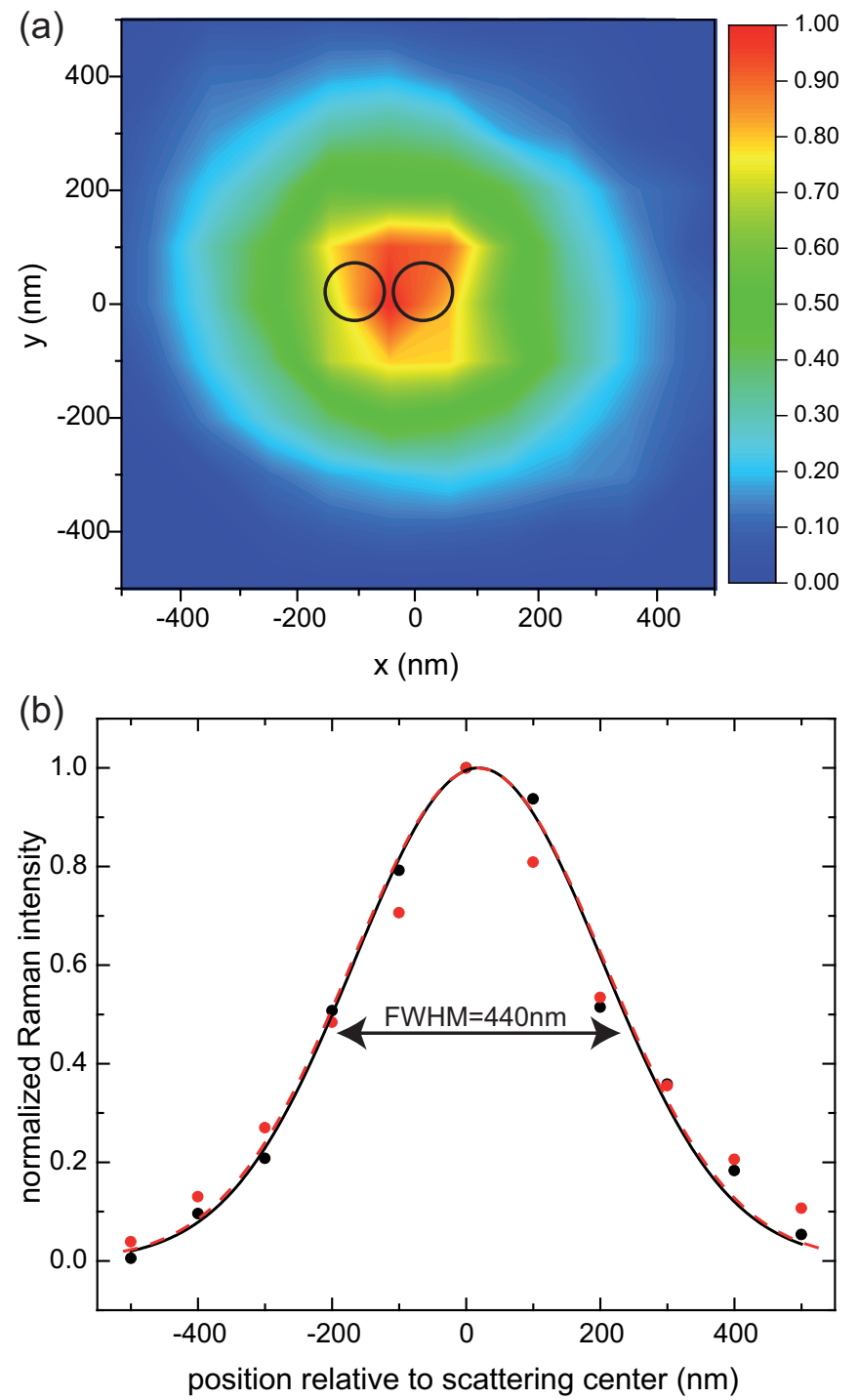

FIG. 6. (a) Raman intensity map of the G mode recorded for a 602$\mathrm{nm}$ excitation wavelength. The dimer is indicated by the two black circles. (b) Intensity measured along the $x$ direction at $y=0$ (small black circles) and along the $y$ direction at $x=0$ (small red circles). Lines are the corresponding fits to a Gaussian intensity profile. The extracted FWHM is $440 \pm 20 \mathrm{~nm}$.

\section{B. Localization of the near-field enhancement}

Plasmonic near-fields localize light to length scales of the order of $10 \mathrm{~nm}$ [37]. The near-fields are challenging to visualize, because most measurements operate with far-field detection techniques. The typical laser spot sizes in a Raman experiment are several hundred nanometers in diameter. Some of us recently introduced a SNOM for inelastically scattered light that measured a SERS hot spot $75 \mathrm{~nm}$ in diameter (lateral size perpendicular to the dimer axis) for gold dimers similar to those studied here [18]. As an alternative approach, we obtain an upper limit for the SERS hot spot by spatially mapping the Raman response of graphene on the dimer in real space.

We scanned an area of $1 \mu \mathrm{m}^{2}$ around the dimer taking Raman spectra every $100 \mathrm{~nm}$. The normalized G-mode intensity is plotted in Fig. 6(a) as a function of the $(x, y)$ position, where 


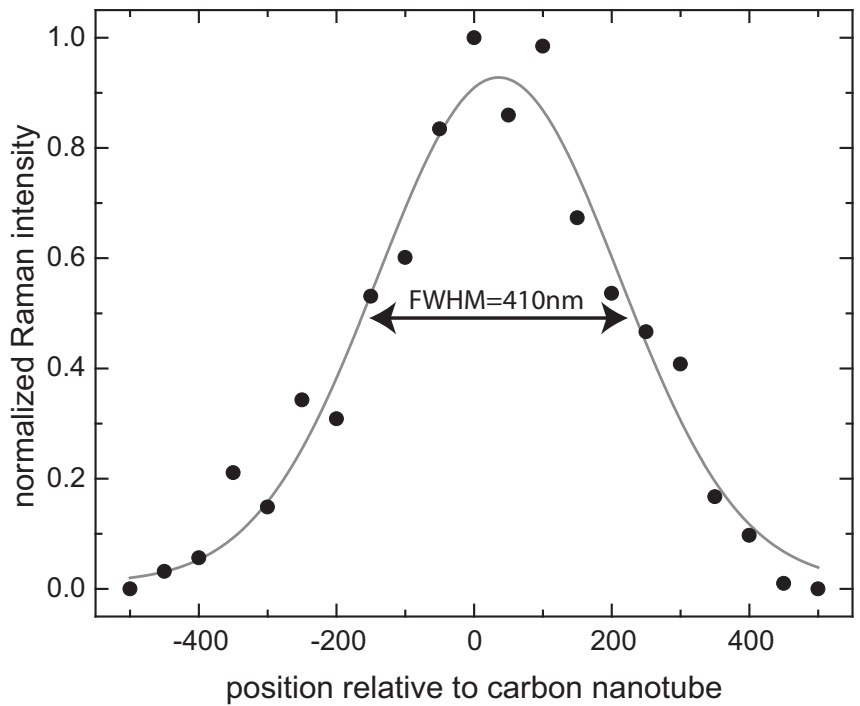

FIG. 7. Determining the laser spot size. Line scan over a small bundle of carbon nanotubes; the G-mode (black circles) intensity is plotted. The Gaussian fit (gray line) gives an FWHM of $410 \pm 30 \mathrm{~nm}$.

the dimer is indicated by the two black circles. This Raman intensity map highlights the localization and spherical shape of the SERS hot spot. Already a shift of $100 \mathrm{~nm}$ results in a decrease in signal intensity of about 10\%; $200 \mathrm{~nm}$ from the center the intensity drops to 50\%. In Fig. 6(b) we plot the intensity of the $\mathrm{G}$ line through the center across the $x$ and $y$ directions. With Gaussian fits we find a full width at half-maximum (FWHM) of $440 \pm 20 \mathrm{~nm}$ in both directions. The behavior reflects a convolution of a localized scatterer with a much more extended laser focus. To measure the diameter of the laser spot we scanned the focus over a small bundle of carbon nanotubes (Fig. 7) and obtained an FWHM of $410 \pm 30 \mathrm{~nm}$. Comparing the width of the Raman map [see Fig. 6(b)] and the diameter of the laser spot we get a width of $160 \pm 95 \mathrm{~nm}$ for the plasmonic hot spot. The width resembles the size of the dimer system. This appears reasonable, because the SERS response accessed by us is dominated by the far-field excitation and detection of the nanodimer. The SNOM-based approach directly excites and detects the near-field of the nanodimer, resulting in the nominally smaller spot size.

The localization of the enhanced signal is also evident in $z$-dependent scans when focusing on the dimer along the $z$ axis, as shown in Fig. 8. Each data point represents the Raman intensity of the 2D mode as a function of the distance between laser focus and probe. Black dots show the normalized signal when focusing on graphene coupled to the dimer, whereas red dots represent the normalized Raman intensity of graphene on the silicon substrate. The trend of both measurements differs strongly. The intensity of the graphene signal on the substrate decreases slowly, whereas the $z$ dependence of the Raman signal on the dimer shows a trend that is expected for a localized scatterer.

The experimental intensities on the dimer should correspond to the point-spread function $s$ in the image plane of the detector, which we calculate with geometric optics [37]. We calculate the point-spread function of a pointlike scattering center that is illuminated by a Gaussian laser beam at a $605-\mathrm{nm}$

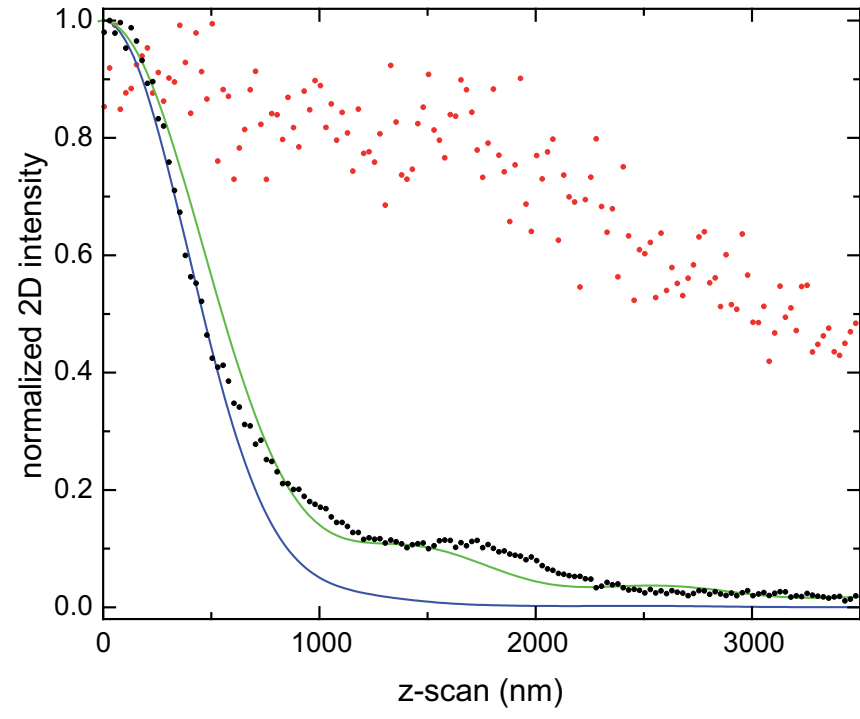

FIG. 8. The $z$ dependence of the 2D-mode intensity of graphene with the laser centered on the dimer (black dots) and on the substrate (red dots). The $z$ scan was recorded at a $605-\mathrm{nm}$ laser wavelength. Solid lines are the calculated detector signal of a pointlike scattering center using geometrical optics and assuming confocal detection (blue curve) or nonconfocal detection (green curve) using Eqs. (1) and (2).

excitation wavelength focused through a $100 \times$ objective with numerical aperture NA $=0.9$. The signal for confocal microscopes (blue line) including both the incident and the detecting pathway is defined as $s_{\text {confocal }} \propto\left|I_{00}\left(\omega_{l}\right) \tilde{I}_{00}\left(\omega_{l}+\omega_{\text {pn2D }}\right)\right|^{2}$, where $\omega_{l}$ is the laser energy and $\omega_{\mathrm{pn} 2 \mathrm{D}}=0.3 \mathrm{eV}$ the phonon energy of the measured 2D mode. $I_{00}$ describes the excitation path [see Eq. (1)], whereas $\tilde{I}_{00}$ describes the detection, Eq. (2). For nonconfocal microscopes (green line) the point-spread function is $s_{\text {nonconfocal }} \propto\left|I_{00}\left(\omega_{l}\right)\right|^{2}$,

$$
\begin{gathered}
I_{00} \propto \int_{0}^{\Theta_{\operatorname{Max}}} f_{w}(\Theta)(\cos \Theta)^{1 / 2} \sin \Theta(1+\cos \Theta) e^{i k_{l} z \cos \Theta} d \Theta, \\
\tilde{I}_{00} \propto \int_{0}^{\Theta_{\operatorname{Max}}}(\cos \Theta)^{1 / 2} \sin \Theta(1+\cos \Theta) e^{i k_{l+\mathrm{pn}} z \cos \Theta} d \Theta,
\end{gathered}
$$

where $\Theta_{\mathrm{Max}}=\arcsin (\mathrm{NA})$ is the maximum collection angle of the objective. $f_{w}=\exp \left(-1 / f_{0}^{2} \mathrm{NA}^{2}\right) \sin ^{2} \Theta$, where $f_{0}=0.8$ is the filling factor of the back aperture of the microscope objective. $k$ is defined as $k=2 \pi / \lambda$ for incident light $k_{l}$ and scattered light $k_{l+\text { pn }}$. The point-spread function was normalized to displacement along $z=0$. The confocal calculation (blue line) resembles very well the measured data until $z=500 \mathrm{~nm}$. The nonconfocal calculation, in contrast, fits better the second maximum at around $z=1700 \mathrm{~nm}$. We conclude that our experimental data represent a combination of confocal detection at closer distances $z$ and nonconfocal detection, when the focus is more distant from the scattering center. We attribute further differences to the simplified model used in the calculation. From the $z$-dependent measurement and the comparison of the laser focus and the Raman map we conclude that the localization is in the order of the size of the plasmonic system. 


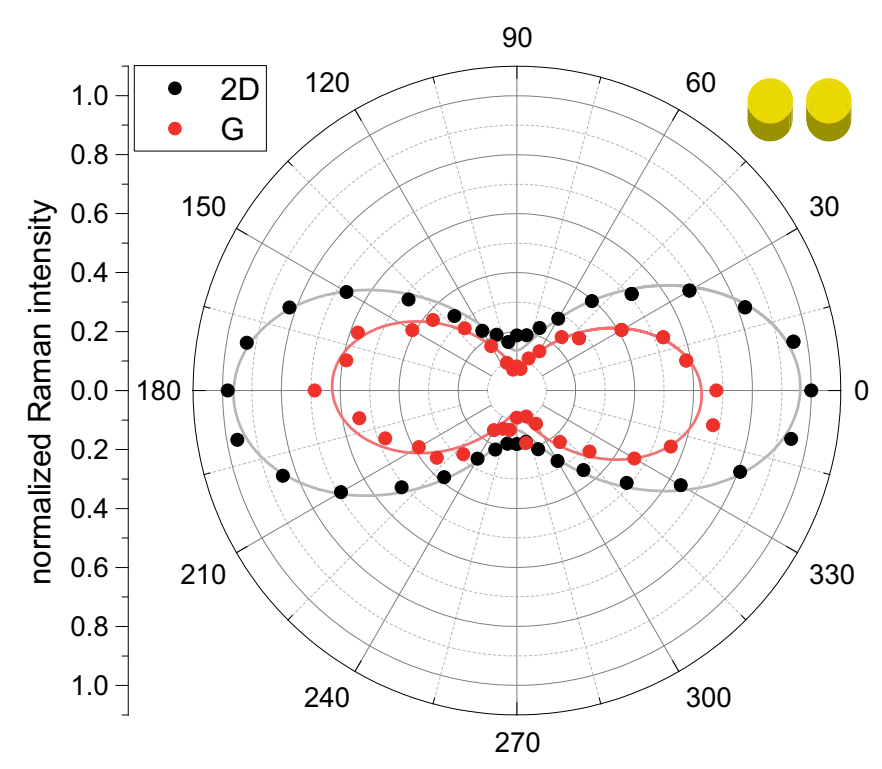

FIG. 9. Polarization-dependent measurements of the G (red circles) and 2D (black circles) modes at an excitation wavelength of $\lambda_{l}=602 \mathrm{~nm}$ when the laser is centered on the plasmonic dimer covered with graphene. The dimer is oriented along the $0^{\circ}-180^{\circ}$ axis as indicated in the inset. Solid lines represent the fits according to Eq. (3).

\section{Polarization dependence of the near-field enhancement}

The Raman intensity of graphene coupled to a plasmonic dimer shows a strong polarization dependence. This was originally demonstrated for two perpendicular polarizations as a proof of the plasmonic enhancement in the dimer-graphene system [20]. Due to the specific design of our canonical SERS system we can switch the plasmonic coupling of the dimer by rotating the incoming polarization. In Fig. 9(a) we show polarization-dependent measurements of the $\mathrm{G}$ and 2D modes. A strong enhancement is observed when the incoming and outgoing polarizations are parallel to the dimer axis. If the polarization is perpendicular to the dimer axis, the Raman intensity decreases by a factor of 10 for the $G$ mode and 6 for the $2 \mathrm{D}$ mode. In perpendicular polarization the particles are decoupled and act as single particles, yielding weaker enhancement [20].

The Raman intensity, in general, is described by $I=$ $\left|\boldsymbol{e}_{i} \cdot R \cdot \boldsymbol{e}_{s}\right|^{2}$, where $\boldsymbol{e}_{i}$ and $\boldsymbol{e}_{s}$ are the polarization direction of the incoming and scattered light, respectively, and $R$ is the Raman tensor [38]. It is sufficient to consider our system to be $2 \mathrm{D}$, because of the nature of graphene, giving a Raman tensor $R=\left(\begin{array}{ll}a & 0 \\ 0 & b\end{array}\right)$. Due to the local near-field configuration of the dimer the components in the $x$ and $y$ directions have to be considered. From the Raman tensor we calculate the Raman intensity as a function of the angle,

$$
I(\Theta)=\left[a \sin ^{2}(\Theta)+b \cos ^{2}(\Theta)\right]^{2},
$$

which is used to fit the experimental data in Fig. 9 with $a_{\mathrm{G}}=0.79$ and $b_{\mathrm{G}}=0.28$ for the $\mathrm{G}$ mode and $a_{2 \mathrm{D}}=0.98$ and $b_{2 \mathrm{D}}=0.37$ for the 2D mode, yielding a similar ratio $a / b$ for both the $\mathrm{G}$ and the $2 \mathrm{D}$ modes. This is reasonable, because

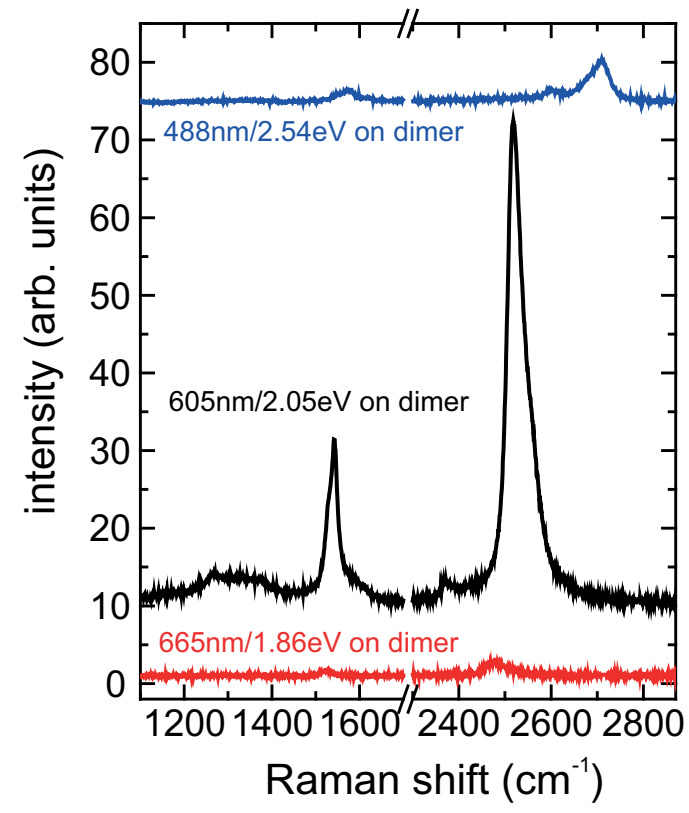

FIG. 10. Spectra of the G and 2D modes on the dimer at different wavelengths: $488 \mathrm{~nm}$ (blue), $605 \mathrm{~nm}$ (black), and $665 \mathrm{~nm}$ (red). Spectra offset for clarity.

the scattering intensity is dominated by the properties of the nanodimer, which is identical for both modes.

\section{Excitation energy dependence}

To investigate and identify the position and width of the plasmonic resonance we measured both graphene Raman modes as a function of the excitation energy in the nearfield and a dark-field spectrum in the far-field. Since pristine graphene has a constant Raman cross section in the visible and near-infrared energy range [23], changes in the Raman intensity as a function of the excitation energy are attributed to the plasmonic near-field. Our experiment directly probes the near-field enhancement of the plasmonic system. Figure 10 shows the enhanced Raman spectra at $488 \mathrm{~nm}$ (blue), $605 \mathrm{~nm}$ (black), and $665 \mathrm{~nm}$ (red), normalized by freestanding graphene (see Sect. II). In the spectrum at $605 \mathrm{~nm}$ a strong enhancement and strain of the graphene modes are observed as discussed above. At 488 and $665 \mathrm{~nm}$ the enhancement decreases strongly; the D mode can no longer be observed.

In the following we present the energy dependence of the near-field enhancement at the plasmonic hot spot. The near-field enhancement was measured at 30 different excitation wavelengths. It is shown in Fig. 11 for the $G$ mode [Fig. 11(a)] and 2D mode [Fig. 11(b)]. Each point represents the integrated area of all enhanced components of both modes. The intensities of the energy-dependent measurements were normalized in the $\mathrm{G}$ or $2 \mathrm{D}$ mode of freestanding graphene directly, giving access to the local near-field enhancement as described in Sec. II. There is a clear maximum in enhancement visible at approximately $2.05 \mathrm{eV}$ for both the $\mathrm{G}$ and the $2 \mathrm{D}$ modes. Almost no enhancement is observed at green excitation (532 nm/2.33 eV). 


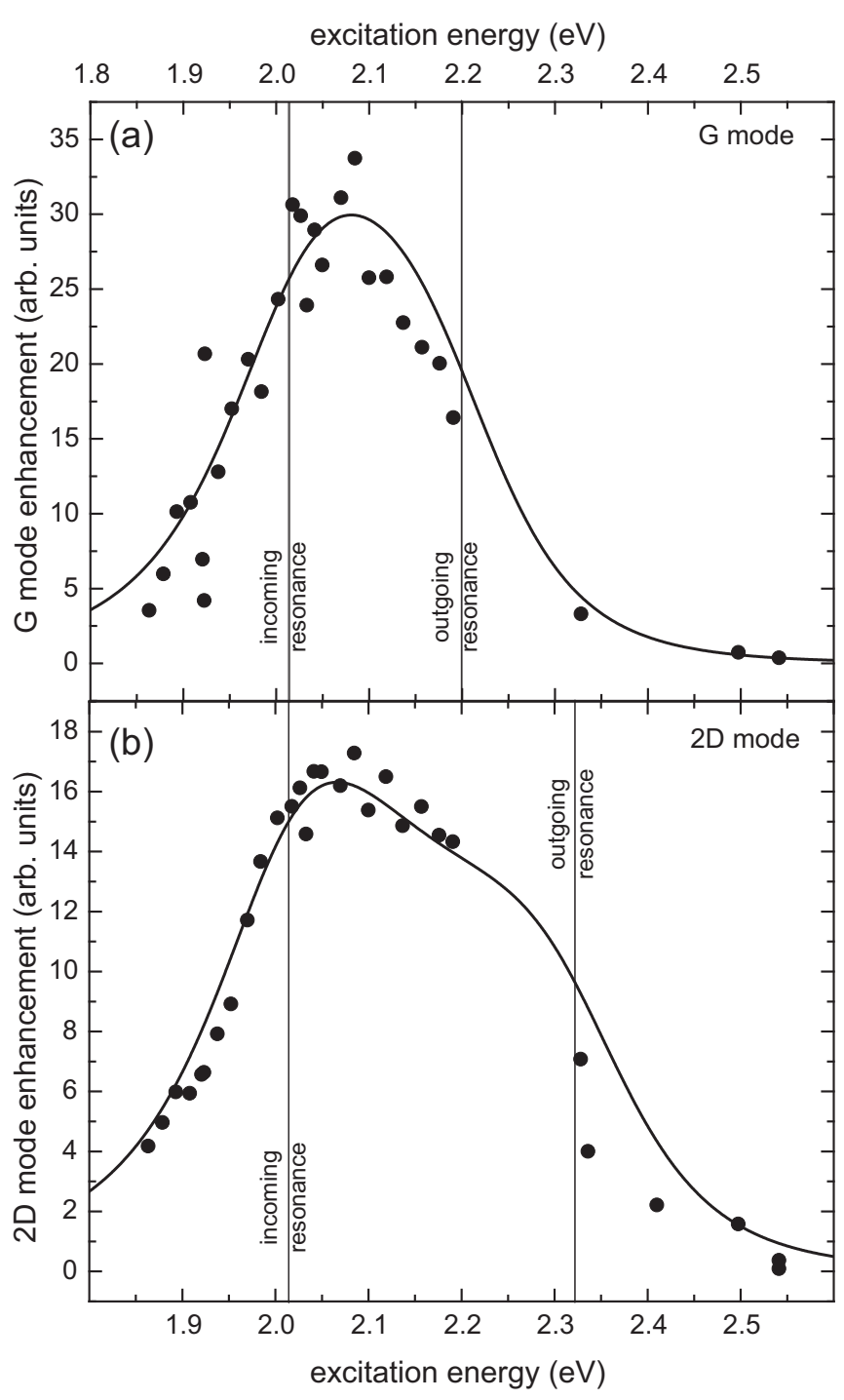

FIG. 11. Energy-dependent measurements of (a) the G and (b) the 2D modes. Solid lines show the fit with higher-order Raman scattering theory [Eq. (4)]. Vertical lines indicate the positions of the incoming and outgoing resonances. The Raman intensity of the $G$ and 2D modes is normalized by suspended graphene as described in Sec. II.

To quantify the energy of the plasmonic resonance from our Raman data we adopt the higher-order Raman scattering description of plasmon-enhanced Raman scattering [14]. It gives the following expression for the enhancement factor $\operatorname{Enh}\left(\omega_{l}\right)$, which we use to fit to the experimental enhancement of the $\mathrm{G}$ and 2D modes in Fig. 11,

$$
\begin{aligned}
\operatorname{Enh}\left(\omega_{l}\right)= & {\left[\frac{M^{2}}{\left(\hbar \omega_{l}-\hbar \omega_{\mathrm{pl}}-\hbar \omega_{\mathrm{pn}}-i \gamma_{\mathrm{pl}}\right)\left(\hbar \omega_{l}-\hbar \omega_{\mathrm{pl}}-i \gamma_{\mathrm{pl}}\right)}\right.} \\
& +\frac{M}{\hbar \omega_{l}-\hbar \omega_{\mathrm{pl}}-\hbar \omega_{\mathrm{pn}}-i \gamma_{\mathrm{pl}}} \\
& \left.+\frac{M}{\hbar \omega_{l}-\hbar \omega_{\mathrm{pl}}-i \gamma_{\mathrm{pl}}}+1\right]^{2}
\end{aligned}
$$

where $M$ is a combined matrix element. The matrix element describes the coupling between the plasmon and the Raman probe [14]. $\omega_{l}$ and $\omega_{\mathrm{pl}}$ are the laser and plasmon frequency, and $\omega_{\mathrm{pn}}$ is the phonon frequency. We use $\omega_{\mathrm{pnG}}=0.19 \mathrm{eV}$ for the strained $\mathrm{G}$ mode and $\omega_{\mathrm{pn} 2 \mathrm{D}} \approx 0.3 \mathrm{eV}$ for the dispersive 2D mode. $\gamma_{\mathrm{pl}}=\hbar / \tau_{\mathrm{pl}}$ is the inverse plasmon lifetime $\tau_{\mathrm{pl}}$, taken to be $0.15 \mathrm{eV}$, which is a common width for a plasmonic system of the dimensions used in this work. Equation (4) describes the interference of four scattering pathways, similar to resonant Raman scattering incorporating the plasmonic coupling. The first term yields the scattering amplitude where the incoming and scattered light interact with the plasmon. The second and third terms give the scattering amplitude when either incoming or scattered light interacts with the plasmon. The last contribution is given by the standard Raman scattering, where no interaction with the plasmon occurs. Since we calculate the enhancement and calibrate on suspended graphene this contribution only gives a constant [14].

The fit of the Raman resonance profile yields a plasmon energy of $\omega_{\mathrm{pl}}=2.01 \mathrm{eV}$ [see Fig. 11(a)]. The excitation energy for maximum enhancement does not correspond to the incoming resonance since the overlap of incoming and outgoing resonance shifts the maximum to higher energies [38]. The predicted enhancement profile of the 2D mode in Fig. 11(b) was obtained from Eq. (4) using the parameters of the G-mode fit but a different coupling $M$. We obtain a good agreement with the experimental data, demonstrating the applicability of our approach. The difference in enhancement for the $G$ and 2D modes is attributed to the different scattering processes of the modes [39]. The energies of the incoming resonances in Fig. 11 are determined by the local near-field resonance, therefore they are identical for the $\mathrm{G}$ and 2D modes. The outgoing resonance, in contrast, originates from the inelastically scattered light and depends on the phonon energy. The asymmetry of the resonance profiles in Fig. 11 results from quantum interference between different scattering processes, which is determined by the sign and magnitude of the combined matrix element $M$. A stronger coupling (described by the $M$ ) implies a lower ratio between incoming and outgoing resonance, approaching unity if the first term in Eq. (4) dominates [14]. Unfortunately we were not able to measure the outgoing resonance of the $G$ or $2 D$ mode in greater detail due to a lack of tunable laser excitation in the respective energy range. We stress once more that the observed resonances originate from the localized near-field enhancement and not from intrinsic resonances of the Raman probe, since graphene has a constant Raman cross section. This emphasizes the suitability of graphene as a probe to investigate the local near-field in plasmonic systems.

The Raman measurements are sensitive to the near-field resonance of the plasmonic system. The far-field response, in contrast, is measured by polarized dark-field spectroscopy. To characterize the plasmon response we obtained a dark-field spectrum of the dimer with the incoming light polarized parallel to the dimer axis (Fig. 12). The dark-field spectrum has a maximum at $2.18 \mathrm{eV}$ and an FWHM of $0.33 \mathrm{eV}$. The maximum of the experimental SERS enhancement occurs at energies lower than the maximum in the dark-field spectrum by roughly $200 \mathrm{meV}$. Differences between elastic scattering (dark-field spectrum) and SERS enhancement are frequently observed in SERS [40]. They are often attributed to (i) an energy difference between the far-field and the near-field resonance and (ii) a distribution in size and shape of plasmonic 


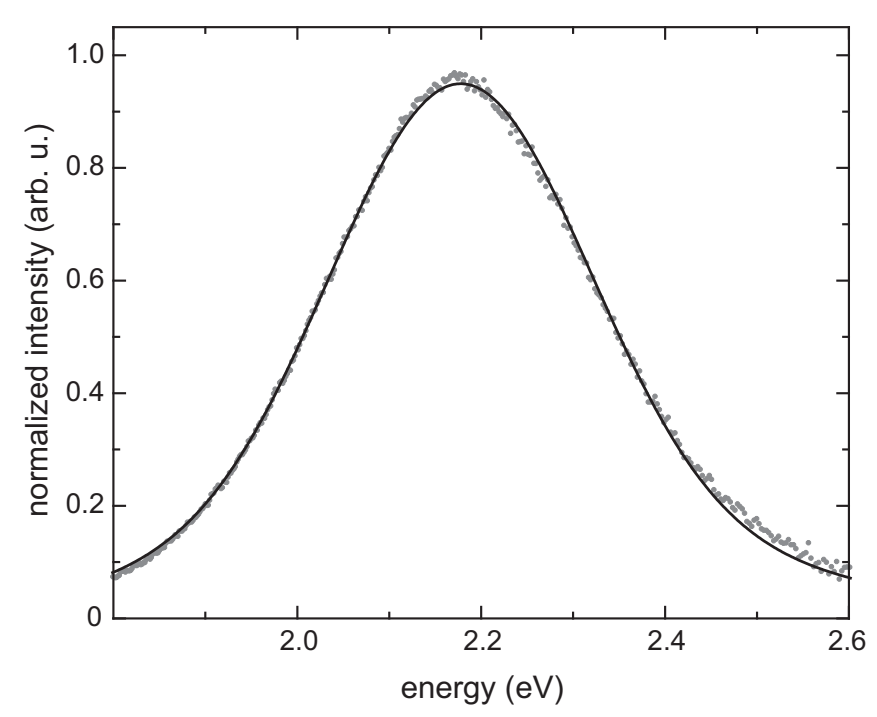

FIG. 12. Normalized dark-field scattering spectrum of the gold dimer with polarization parallel to the dimer axis. The solid line shows a Gaussian fit of the experimental data (gray points).

hot spots in colloidal samples that differs for elastic and inelastic plasmon-enhanced scattering [41] Since our system contains only a single plasmonic hot spot, explanation (ii) does not apply. We now examine explanation (i): Plasmon-enhanced Raman scattering yielded a near-field resonance of $\omega_{\mathrm{pl}}=$ $2.01 \pm 0.02 \mathrm{eV}$ (Fig. 11), whereas the far-field resonance occurred at $2.18 \pm 0.01 \mathrm{eV}$. Following Ref. [40] we calculated an expected shift between far- and near-field of $0.04 \mathrm{eV}$ using the model of a damped oscillator and the geometry of our nanodimer. The predicted shift is much smaller than that measured experimentally $(0.17 \mathrm{eV})$. We could not identify the origin of this surprisingly large shift, which will be investigated in more detail in the future. One contribution may arise from the substrate and the varying dielectric environment or the strongly damping adhesion layer between the substrate and the gold.

\section{CONCLUSION}

In summary, we have shown that graphene can be used as a local Raman probe to analyze the near-field properties of a plasmonic gold nanodimer system. Due to adhesion forces from the substrate the suspended graphene is pulled into the plasmonic hot spot at the dimer gap. We were able to determine the local hydrostatic strain of the graphene to be $2.1 \%$ and the local shear strain to be $1.1 \%$. We measured an n-type doping level of $10^{13} \mathrm{~cm}^{-2}$. The localization of the plasmonic hot spot of the dimer was confirmed by a spatial Raman map and measuring the Raman intensity dependent on the distance from the laser focus to the dimer.

We investigated the local near-field properties of the plasmonic hot spot. We observe a strong polarization dependence of the Raman intensity on the nanodimer. If the polarization is chosen along the dimer axis, strong enhancement is observed; for perpendicular polarization the intensity drops by an order of magnitude. We measured a near-field resonance at $2.01 \mathrm{eV}$. We quantitatively compared the energetic position of the near-field (Raman) and far-field (dark field: $2.18 \mathrm{eV}$ ) resonance of the plasmonic system.

Since the investigated system consists of a well-defined plasmonic nanostructure and a Raman probe with well-known properties it is an excellent candidate for investigation of the fundamental coupling mechanism in surface-enhanced Raman scattering. Graphene may also be used as a reference in SERS studies to quantify the enhancement factor of a given substrate or nanostructure.

\section{ACKNOWLEDGMENTS}

This work was supported by SFB 658, subproject A6, and the Focus Area NanoScale of Freie Unitversität Berlin. N.S.M. thanks Deutsche Telekom Stiftung for financial support. N.C. acknowledges support from the North West Nanoscience (NowNano) DTC Grant EP/G03737X/1. F.S. acknowledges funding from the European Graphene Flagship Project and the ERC Synergy grant Hetero2D.
[1] P. L. Stiles, J. A. Dieringer, N. C. Shah, and R. P. Van Duyne, Annu. Rev. Anal. Chem. 1, 601 (2008).

[2] E. C. Le Ru and P. G. Etchegoin, Annu. Rev. Phys. Chem. 63, 65 (2012).

[3] H. Xu, J. Aizpurua, M. Käll, and P. Apell, Phys. Rev. E 62, 4318 (2000).

[4] S. L. Kleinman, E. Ringe, N. Valley, K. L. Wustholz, E. Phillips, K. A. Scheidt, G. C. Schatz, and R. P. Van Duyne, J. Am. Chem. Soc. 133, 4115 (2011).

[5] M. Fleischmann, P. J. Hendra, and A. J. McQuillan, Chem. Phys. Lett. 26, 163 (1974).

[6] D. L. Jeanmaire and R. P. Van Duyne, J. Electroanal. Chem. Interfac. Electrochem. 84, 1 (1977).

[7] C. L. Haynes and R. P. Van Duyne, J. Phys. Chem. B 107, 7426 (2003).

[8] E. Petryayeva and U. J. Krull, Anal. Chim. Acta 706, 8 (2011).

[9] Q.-1. Li, B.-w. Li, and Y.-q. Wang, RSC Adv. 3, 13015 (2013).
[10] B. Sharma, R. R. Frontiera, A.-I. Henry, E. Ringe, and R. P. Van Duyne, Mater. Today 15, 16 (2012).

[11] G. C. Schatz, M. A. Young, and R. P. Van Duyne, in SurfaceEnhanced Raman Scattering (Springer, Berlin, 2006).

[12] E. Ru and P. Etchegoin, Principles of Surface-Enhanced Raman Spectroscopy: And Related Plasmonic Effects (Elsevier Science, Amsterdam, 2008).

[13] P. Roelli, C. Galland, N. Piro, and T. J. Kippenberg, Nat. Nanotechnol. 11, 164 (2015).

[14] N. S. Mueller, S. Heeg, and S. Reich, Phys. Rev. A 94, 023813 (2016).

[15] N. Mirsaleh-Kohan, V. Iberi, P. D. Simmons, N. W. Bigelow, A. Vaschillo, M. M. Rowland, M. D. Best, S. J. Pennycook, D. J. Masiello, B. S. Guiton, and J. P. Camden, J. Phys. Chem. Lett. 3, 2303 (2012).

[16] R. Esteban, R. Vogelgesang, J. Dorfmüller, A. Dmitriev, C. Rockstuhl, C. Etrich, and K. Kern, Nano Lett. 8, 3155 (2008). 
[17] R. Hillenbrand, F. Keilmann, P. Hanarp, D. S. Sutherland, and J. Aizpurua, Appl. Phys. Lett. 83, 368 (2003).

[18] P. Kusch, S. Mastel, N. S. Mueller, N. M. Azpiazu, S. Heeg, R. Gorbachev, F. Schedin, U. Hübner, J. I. Pascual, S. Reich, and R. Hillenbrand, Nano Lett. 17, 2667 (2017).

[19] T. Sannomiya, J. Junesch, and P. Rajendran, J. Opt. Res. 15, 73 (2013).

[20] S. Heeg, R. Fernandez-Garcia, A. Oikonomou, F. Schedin, R. Narula, S. A. Maier, A. Vijayaraghavan, and S. Reich, Nano Lett. 13, 301 (2013).

[21] S. Heeg, A. Oikonomou, R. F. Garcia, S. A. Maier, A. Vijayaraghavan, and S. Reich, Physica Status Solidi Rapid Res. Lett. 7, 1067 (2013).

[22] N. S. Mueller, S. Heeg, M. P. Alvarez, P. Kusch, S. Wasserroth, N. Clark, F. Schedin, J. Parthenios, K. Papagelis, C. Galiotis, M. Kalbac, A. Vijayaraghavan, U. Huebner, R. Gorbachev, O. Frank, and S. Reich, 2D Mater. 5, 015016 (2017).

[23] P. Klar, E. Lidorikis, A. Eckmann, I. A. Verzhbitskiy, A. C. Ferrari, and C. Casiraghi, Phys. Rev. B 87, 205435 (2013).

[24] L. M. Malard, M. A. Pimenta, G. Dresselhaus, and M. S. Dresselhaus, Phys. Rep. 473, 51 (2009).

[25] T. M. G. Mohiuddin, A. Lombardo, R. R. Nair, A. Bonetti, G. Savini, R. Jalil, N. Bonini, D. M. Basko, C. Galiotis, N. Marzari, K. S. Novoselov, A. K. Geim, and A. C. Ferrari, Phys. Rev. B 79, 205433 (2009).

[26] K. S. Novoselov, A. K. Geim, S. V. Morozov, D. Jiang, Y. Zhang, S. Dubonos, I. V. Grigorieva, and A. A. Firsov, Science 306, 666 (2004).

[27] A. Reina, H. Son, L. Jiao, B. Fan, M. Dresselhaus, Z. Liu, and J. Kong, J. Phys. Chem. C 112, 17741 (2008).

[28] A. C. Ferrari and D. M. Basko, Nat. Nanotechnol. 8, 235 (2013).
[29] A dye laser with different fluorescent dyes was used to excite the scattering process in the visible $(560-680 \mathrm{~nm})$; further, some lines (488-568 nm) of an $\mathrm{Ar} / \mathrm{Kr}$ laser were used. For the excitation at $532 \mathrm{~nm}$ a Nd:YAG laser was used.

[30] D. Yoon, H. Moon, Y.-W. Son, J. S. Choi, B. H. Park, Y. H. Cha, Y. D. Kim, and H. Cheong, Phys. Rev. B 80, 125422 (2009).

[31] K. Ikeda, M. Takase, N. Hayazawa, S. Kawata, K. Murakoshi, and K. Uosaki, J. Am. Chem. Soc. 135, 11489 (2013).

[32] F. Benz, M. K. Schmidt, A. Dreismann, R. Chikkaraddy, Y. Zhang, A. Demetriadou, C. Carnegie, H. Ohadi, B. d. Nijs, R. Esteban, J. Aizpurua, and J. J. Baumberg, Science 354, 726 (2016).

[33] M. Lazzeri and F. Mauri, Phys. Rev. Lett. 97, 266407 (2006).

[34] S. Huh, J. Park, K. S. Kim, B. H. Hong, and S. B. Kim, ACS Nano 5, 3639 (2011).

[35] Z. Fang, Y. Wang, Z. Liu, A. Schlather, P. M. Ajayan, F. H. L. Koppens, P. Nordlander, and N. J. Halas, ACS Nano 6, 10222 (2012).

[36] A. Das, S. Pisana, B. Chakraborty, S. Piscanec, S. K. Saha, U. V. Waghmare, K. S. Novoselov, H. Krishnamurthy, A. K. Geim, A. C. Ferrari, and A. K. Sood, Nat. Nanotechnol. 3, 210 (2008).

[37] L. Novotny and B. Hecht, Principles of Nano-Optics (Cambridge University Press, Cambridge, UK, 2006).

[38] M. Cardona, R. K. Chang, G. Güntherodt, M. B. Long, and H. Vogt, Light Scattering in Solids II, edited by M. Cardona and G. Güntherodt (Springer-Verlag, Berlin, 1982).

[39] C. Thomsen and S. Reich, Phys. Rev. Lett. 85, 5214 (2000).

[40] J. Zuloaga and P. Nordlander, Nano Lett. 11, 1280 (2011).

[41] K. L. Kelly, E. Coronado, L. L. Zhao, and G. C. Schatz, J. Phys. Chem. B 107, 668 (2003). 